\title{
DIE ERSTELLUNG EINER ANIMATION ALS LERNMEDIUM FÜR DIE GRAMMATIK IM DEUTSCHUNTERRICHT
}

\author{
Vopy Endang Lestari \\ Ahmad BengarHarahap \\ Hafniati
}

\begin{abstract}
AUSZUG
.Das Ziel dieser Untersuchung ist es, um eineAnimationalsLernmediumfür die Grammatik imDeutschunterrichtzu erstellen. Die Erstellungsuntersuchung werden in dieserUntersuchungangewendet.

Der

Prozess

der

ErstellungdemLernmediumverwendet Borg und Gall Theorie. Die ErstellungsphasebestehtausfünfPhasen. Die Phasensind (1) die Untersuchung und Sammlung von Information, (2) die Planung, (3) die Erstellung des Pdodukts, (4) die Validierung von Experten, (5) der Revision des Produkts. In dieserUntersuchungwurdeeinneuesLernmediumerstellt.Die Daten dieser Untersuchung ist die grammatikalischen Regeln zum Akkusativ oder Dativ bei Präpositionen.Die Datenquelle dieser Untersuchung kommtaus dem Buch "Grammatik-ABC für Deutsch als Fremdsprachen auf Zertifikatsniveau und Niveaustufen A1, A2, B1, B2" von Franz Eppert. Die Ergebnis der ErstellungeineAnimationalsLernmediumfür die Grammatik imDeutschunterrichtist die Videoanimationfür die PräpositionenimAkkusativ und Dativmit den BärenalsVisuellefigur, denn der Bär ist ein Symbol des Landes von Berlin und ein lustiges Tier. Die Animation wird in Video hargestellt und dauert 05.41 Minuten. Nach der Erstellungwurde die Lernmedienbei der Expertenvalidiertoderüberprüft. Basierend auf der Valiedierungist die Lernmedien gut.Durch die Ergebnisse der Untersuchungwirderwartet, um Kenntnisseüber die Grammatik fürAnfängerinteressanterzuverstehen.
\end{abstract}

Schlüsselwörter:Die Animation, die Grammatik.

\section{EINLEITUNG}

Die Lernmedien im Allgemeinen sind ein Mittel des Lernprozesses. Die Lernmedien sind das physische Mittel, um die Inhalte wie Bücher, Filme, Videos und so weiter zu vermitteln. In der Ausbildung können verschiedene Lernmethode eingesetzt werden. Eine Lernmethode, die jetzt entwickelt werden kann, kann die Computer-Technologie nutzen. Die Computertechnologie kann Lernmaterialien wie Texte, Audios und Video liefern. Die Audio-visuellen-Medien können in Form von interaktiven Anwendungen wie Animationen benutzt werden.

Die Animation in der Ausbildung fungiert als Lernmedium, das interessant ist. Die Animation ist eine Form der visuellen Bewegungen, die benutzt werden kann, um 
schwierige Lerninhalte zu erklären. Nach Vaughan (in Binanto 2010:219) ist die Animation ein Versuch, eine statische Präsentation zum Leben zu erwecken. Die Animation ist visuelle Veränderungen im Laufe der Zeit, die große Macht auf Multimedia-Projekte und Web-Seiten erstellt werden. Die Animationen sind Bilder, die auf eine bestimmte Art Weise, mit einer bestimmten Geschwindigkeit und Richtung bewegt werden. Eine Animation erklärtdie Wirkung der Motion-Effekt oder die Verformungswirkung. Die Animation ist auch eine Technik, die Bilder nacheinander so anzeigt, dass das Publikum die Illustrationenbewegung auf dem Bild fühlt. Die Animation wird verwendet, um die Bewegung eines Objekts zu beschreiben. Die Animation in der Ausbildung fungiert als interessantes Lernmedium. Die Animation ist eine Form der visuellen Bewegung, die verwendet werden können, um schwierige Gegenstände zu erklären.

Im Deutschunterricht können Animation als Lernmedium verwendet werden, wenn man die Grammatik lernt. PräpositionenAkkusativ und Dativisteinschwieriges Material beim Grammatik zulernen. Die Deutschlernende sindoft fälschlich und sie sind schwierig, die Einsatz von Präpositionen Akkusativ und Dativ zu unterscheiden.Viele Deutschlernendehaben noch Schwierigkeiten, die Grammatik zu verstehen, denn die Lernmedien sind uninteressant. Mithilfe von Animation werden die Deutschlernende eher zum Lernen der Grammatik angeregt und die Grammatik kann leichter verstanden werden. Basierend auf den obengenanntenProblemenimDeutschunterrichtwird"Die Erstellungeiner Animation alsLernmediumfür die Grammatik imDeutschunterricht".

\section{THEORETISCHE GRUNDLAGE}

\section{Der Begriff der Animation}

Brugger (in Heller 2004:8) meint, dass die Animation vom lateinischen Wort „animire“ abgeleitet wird und übersetzt „,belebt“ oder „,bewegt“ bedeutet. Animation heißt also einem Objekt Leben zu geben, was sich nicht selber bewegen kann. Der Begriff Animation wird in der Praxis nur für Laufbild-Sequenzen genutzt, also aufgezeichnete Einzelbilder von angelegten Bewegungsabläufen statischer Objekte oder Zeichnungen, die mit erhöher Geschwindigkeit abgespielt von dem menschlichen Auge als Bewegung wahrgenommen werden.

Nach Vaughan (in Binanto 2010:219) ist die Animation ein Versuch, eine statische Präsentation zum Leben zu erwecken. Die Animation ist visuelle Veränderungen die ganze Zeit, die große Macht auf Multimedia-Projekte und Web- 
Seiten geben.Saeba (2008: 223) stellt fest, dass die Animation im Grunde eine Sammlung von aneinandergereihten Bildern ist. Die Bilder werden der Reihe nach in einer relativ schnellen Zeit angezeigt. Laut dem Konzept der Animation ist jedes Bild ein Frames. (vgl. Bunadi und Zeembry 2007: 9).

\section{Die Arten der Animation}

Bei der Entwicklung der Animation als Lernmedium wird Animationsart "Puppenanimation" benutzt.Puppenanimation ist eine interessante Animationsart. Mit Puppenanimation gibt es viele Charakteren, wie Tiere, Pflanzen usw, die gemacht werden können. Nach Partmore (in Binanto 2010: 223) gibt es mehrere Arten von Animationen, nämlich: Stop-Motion, Zell Animation, Zeitraffer (Time-Lapse), Claymation, Cut-Out-Animation und Puppenanimation.

\section{Der Begriff der Lernmedien}

Sadiman (in Barus und Suratno 2015: 17) erklärt, dass die Medien eine Software sind, die die Nachrichten oder Bildungsinformationen präsentieren. Criticos (in Daryanto 2010: 4) meint, dass die Medien eines der Kommunikationsmittel sind, die eine Nachricht vom Kommunikator zum Kommunikant übermitteln. Basierend auf diesen Definitionen kann gesagt werden, dass die Lernmedien Vermittler im Lernprozesse sein.Das Wort Medien kommt vom lateinischen Wort „Medium“, das bedeutet wörtlich Mittler, intermediär oder Einführung. Die Lernmedien spielen eine wichtige Rolle im Lernprozess. Die Lernmedien werden als Instrumentvon den Lehrernbenutzt, um die Deutschlernende im Unterricht zu motivieren.

Barus und Suratno (2015: 17) erklären, dass die Lernmedien alle Mittel oder Gegenstände sind, die während der Lernaktivitäten mit der Absicht verwendet werden, um eine Lerninformation von der Quelle (dem Lehrer) an den Empfänger (die Lernenden ) zu vermitteln. Im Lernprozess sind die Medien sehr wichtig, denn sie können den Vermittlungprozess unterstützen. Das Medium ist ein Mittel, das es dem Lehrer erleichtert, Kursmaterial $\mathrm{zu}$ finden und $\mathrm{zu}$ vermitteln und das den Schülern beim Lernen helfen kann, neuen Stoff zu verstehen. 
Basierend auf einigen Expertenmeinungen kann zusammengefasst werden, dassdie Lernmedien alle Mittel und Materialien sind, die hifsbereich im Unterricht machen können. Moderne Lernmedien können also ein effektiveres, effezienteres und interessanteres Lernen ermöglichen.

\section{Die Arten der Lernmedien}

Nach Ankerstein (in Bausch, et al 2007:396) sind visuelle Medien in der Fachliteratur sowohl Tafelzeichnungen und Buchillustrationen als auch Diaprojektionen oder Stummfilme. Audiovisuelle Medien sind Unterrichtsmittel, bei denen Bild und Ton als integrierte Bestandteile einer Informationsvermittlung auftreten, zum Beispiel bei einer Fernsehsendung oder einer Filmvorführung. Auf dieser Grundlage entstanden um die Mitte des vergangenen Jahrhunderts herum audiovisuelle Lehrwerke, die im Rahmen einer audiovisullen Methode zum Einsatz gelangten (Schiffler, Schilder (in Bausch, et al 2007:396)). Im Unterrichtsalltag der Schule blieb die Verwendung solcher Lehrwerke jedoch auf wenige Ausnahmen, meist im Zusammenhang mit Modellversuchen beschräkt (Strack in Bausch, et al 2007:396), weil sie das reproduktive Lernen einseitig förderten und das kreative Potenzial der Schüler nicht forderten.

\section{Der Begriff "Grammatiken"}

Crystal (in Fauziah 2014:2) hat gesagt, dass die Grammatik die Regekn der Satzstruktur basierend auf Sintax und Morphologie ist. Nach Zimmermann (in Bausch, et al 2007:406) warden unter Grammatiken Texte über die phonologischen, morphologischen, syntaktischen, textuellen und semantisch-pragmatischen Eigenschaften einer Sprache (oder mehr als einer Sprache, z.B. bei sprachvergleichenden Grammatiken) verstanden. Nicht jede Grammatik berücksichtigt alle diese Eigenschaften. Mit Texten sind neben Büchern, grammatischen Beiheften usw. auch digital gespeicherte Informationen gemeint.

\section{Präpositionen}

Stang, et.al (2014:205) erklärt, dass die Präpositionen ihrer Formnach unveränderlich sind. Sie treten immer mit einem anderen Wort, in der Regel einem Substantiv oder Pronomen, auf, dessen Fall sie bestimmen $(>>$ regieren $<<)$. Viele Präpositionen können auch zwei Fälle $>>$ regieren $<<$. Präpositionen stehen meist vor 
dem regierten Wort. Die Präpositionen haben viele Typen, nämlich: Akkusativ, Dativ, Akkusativ-Dativ und Genetiv.

\section{Macromedia Flash 8}

Nach Wahyono (2006:1) ist Macromedia Flash 8 ein Medium, das verwendet wird, um die Gestaltung und Präsentationsvorrichtung, Veröffentlichungen oder andere Anwendung zu machen,die die Verfügbarkeit von Mitteln zur Interaktion mit den Nutzern erfordern. Die erstellten Projekte mit Flash sind Text, Bilder, einfache Animationen, Videos oder andere Spezialeffekte.

\section{Die konzeptuelle Grundlage}

Die Grammatikvermittlung ist ein schwieriges Thema im Deutschunterricht. Um Grammatik leicht lernen zu können, müssen spannende Lernmedien verwendet werden. In diesem Fall können Animation als Lernmedien hilfreich sein. Da in der Animation gibt es Bilder, die innovativ sind, interessante Stimme, Bewegungen Mittel empfehlenswert und Songs sind Spaß. So können etwa komplexe Sachverhalte visuell und dynamisch beim schwierige Grammatik einfach darstellen. Durch die Verwendung von Animation als Lernmedien wird die Deutschlernende interessierter und motivierter, um Grammatik zu lernen. Sie können auch einfacher die Grammatik verstehen. Zusätzlich wollen die Lehrer auch leichter, wenn die Grammatik der Lernmaterialien mit Animation als Lernmedium erklären.

\section{UNTERSUCHUNGSMETHODE}

Diese Untersuchung ist eine Erstellungsuntersuchung. Es wird ein bestimmtes Produkt erstellt und dann auf seine Wirksamkeit hin getestet.

Diese Untersuchung wird in der Klasse der Deutschabteilung an der staatlichen Universität von Medan durchgeführt.

Diese Erstellungsuntersuchung ist die Entwicklung einer Animation zum Thema "Die Grammatik über Präpositionen mit dem Akkusativ oder dem Dativ (wo oder wohin)", wobei das Erstelllungsmodell von Borg \& Gall (1983:775) verwendet wird. Die Schritte dieses Erstellungsmodell sind folgenden:

1. Die Untersuchung und Sammlung von Information

2. Die Planung 
3. Die Erstellung des Produkts

4. Die Validierung von Experten

5. Der Revision des Produkts

6. Die Testen des Hauptfeldes

7. Das Revisionprozess

8. Die Testen des OperationalenFeldes

9. Die Revision des Produkts

10. Die Verbreitung und Umsetzung

In dieserUntersuchung, wird die SchrittemitdemNummer (1), (2), (3), (4) und (5) anwenden. ImFolgendenwerden die Schritte des Entwicklungsmodells von Borg und Gall erklärt.

1) Die Informationssammlung

In dieser Phase wird die Umfrageoderein Interview gemacht. Die UmfrageenthältInformationen, $\quad$ ob dieDeutschlernende die schwierigeGrammatik beimLernen hat odernicht, ob die Lehrer eininteressantesMedienbenutztodernicht, wenn die Grammatik lernen.

2) Die Planung

Die nächste Phase ist die Planung. ZuerstwirdAnlyse das Material gemacht, um die schwierigeGrammatikvermittlungzuwissen, dannwird die interessantesSätzegeschriebt, um im Animation zumachen. In dieser Phase wird das Konzeptgemacht, zumBeispiel das Konzept des Design der Animation sowiefürSatz, Thema, Hintergrund, Bilder, Stimme und Musik.Mit den Ergebnissen der Analysekann die Herstellung von Animation alsLernmediumgeplantwerden.

3) Die Erstellung des Produkts

Nachdem die Datengesammeltwerden, werdensie die Erstellung der einerAnimation im Macromedia Flash 8 verwendetwerdensollen, vorbereitet. Danachwird das LernmediumzumThema "PräpositionenAkkusativ und Dativ" erstellt. Das hierverwendete material hat das Thema "die Grammatik überPräposition (wooderwohin)". Durch die Puppenanimationkann eine Vielzahl von Charakterenbestehen, zum BeispielTiercharakteren. Der Bär wird als ein Tiercharakter benutzt, denn der Bär ist ein Symbol des Landes von Berlin und ein lustiges Tier. Der Bär kann auch das Interesse der Deuschlernende beim Lernen von Grammatik anziehen. In dieser Animation 
wird der Bär als Charakter der Menschen gebildet. Das Konzept, das in der zweite Phase geplant wird, wird in dieser Phase benutzt.

4) Die ValidierungdurchExperten

In diesemSchrittwird die Animation bewertet. Dannwerden die Validierunggemacht. Man sollkorrigiertwerden, um die Animation alsLernmediumschon gut odernichtzuwissen. Nach der Validierungwird die Animation alsLernmediumverbessert. Diese Animation alsLernmediumwird von Expertengeprüft. Die ExpertensindDozenten und deutsche Muttersprachler.

5) Der Revision des Produkts

Nachdem die Experten die Validierunggemachthaben, wird die Animation alsLernmediummit den Umfragenverbessert.

\section{ERGEBNIS DER UNTERSUCHUNG}

Dieses Kapitel handelt von den Untersuchungsergebnissen. In diesem Kapitel werden der Prozess und die Ergebnisse der Erstellungeiner Animation alsLernmediumfür die Grammatik imDeutschuterricht erklärt. Die Schritte und die Ergebnisse der Untersuchung werden in diesem Kapitel erklärt.

\section{Der Prozess der Erstellung}

In dieser Untersuchung wird das Untersuchungsmodell von Borg und Gall benutz. Dieses Modell besteht aus fünf Phasen, nämlich (1) die Untersuchung und Sammlung von Information, (2) die Planung, (3) die Erstellung des Pdodukts, (4) Validierung von Experten, (5) der Revision des Produkts.In diesem Kapitel wird jeder Schritte mit dem Entsprechenden Ergebnis Skizziest.

1. Die Untersuchung und Sammlung von Information

Dies ist die erste Phase um die Animation alsLernmediumfür die Grammatik zuerstellen. In dieser Phase der Beobachtung werden Informationen über das Lernen der Deutschlernenden, ihrer Bedürfnisse und die Lernmedien im Deutschunterricht gesammelt.Die Bedürfnisse den Schülern ist, was die Medien nicht finden, dass es als eine Quelle des Lernens der Schüler beim Deutschunterricht in der Schule verwendet. Das Medium, das im Lernprozess verwendet, normalerweisenur das Buchlesen, Präsentationmedien oder Web-Lernen und Lernbedingungen. 


\section{Die Planung}

In dieser zweiten Phase wird die Plannung durchgefürht, manerhälteinen Überblick über die AnimationalsLernmedium, die entwickelt werden kann, um vorgeschriebenes Material, wiebeispielsweisedas Material"PräpositionenAkkusativ und Dativ" imDeutschunterrichtzuverwenden. In dieser Phase wird das Konzept gemacht, nämlich: Satz, Thema, Hintergrund, Bilder, Stimme und Musik. In dieser Phase wird das Konzept der Animation mithilfe Macromedia Flash 8 erstellt.

a. Die Bilder

Dieses animierte Video benutztBären als visuelle Figur.Der Bärlebt in einem Wald, der kühl und bequemist und mitschattigen und grünenBäumengefülltist. Denn der Bär ist ein Symbol des Landes von Berlinund ein lustiges Tier. Der Bär wird erwartet, um das Interesse der Deutschlernenden beim Lernen von Grammatik wecken zu können.

b. Der Hintergrund

Bei der Hintergrundfarbe dominiert Braun, da die braune Farbe identisch ist mit der Bärenfarbe. Die braune Farbe ist auch eine geeignete Farbe, die zu anderen Farben passt.

c. Der Satz „Präpositionen im Akkusativ und Dativ“

d. Die Stimme

Die Stimme wird von einem Muttersprachler aus Deutschland gesprochen. Deutschlernende können so nicht nur Grammatik lernen, sondern auch jedes Wort, das sie hören, richtig aussprechen.

e. DerMusikbestimmen

Das Lied, welches in diesem Video verwendetwird, isteinLied ausYoutube. Das Lied wurde ausgewähltund so angepasst, dass die Animation lebendiger wird. Die Musikinstrumente sindklassische deutsche Instrumente. Diese Musik kann die Darstellung von Lernmedien interessanter machen. Außerdem werden Deutschlernende unter Begleitung der Musik das Material erwartet, umzu verstehen und die Musik der deutschen Klassik genießen.

f. Das Thema

Das Thema dieses animierten Video ist Präpositionen Akkusativ und Dativ. Für dieses Thema wurde der Bär als Hauptdarsteller ausgewählt, um jede Bewegung aus dem Beispiel des Satzes "Präpositionen Akkusativ und Dativ" auszuführen. Der Bär ist ein Symbol des Landes von Berlin und ein lustiges 
Tier, das Interesse des Betrachters wecken kann. Mit diesem Thema werden die Deutschlernenden begeisterter, die Grammatik Praepositionen Akkusativ und Dativ zu lernen. Sie können es auch leichter verstehen.

\section{Der Ergebniss des Produkts}

Das Ergebnis der Untersuchung ist eine Videoanimation mit der Anwendung Macromedia Flash 8. Diese Videoanimation dauert fünf Minuten 41 Sekunden und die Datei umfasst 782 MB (Megabyte). Die Videoanimation kann mithilfe des GOM Players und $V L C$ geöffnet werden. Unten werden einige der Ergebnisse des Designs des Lernmediums erklärt.

a) Die Einbände des Lernmediums

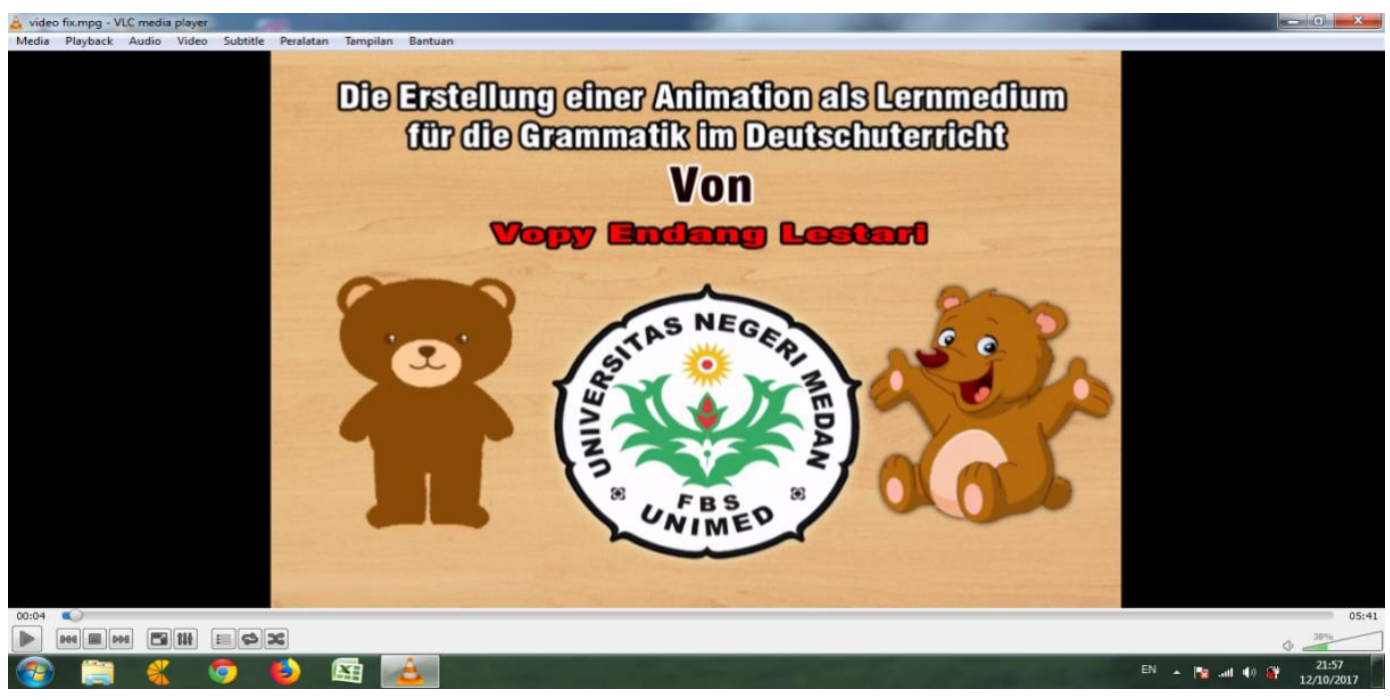

b) Ein Beispiel aus dem Ergebnis für das Wort Präpositionen Akkusativ und Dativ
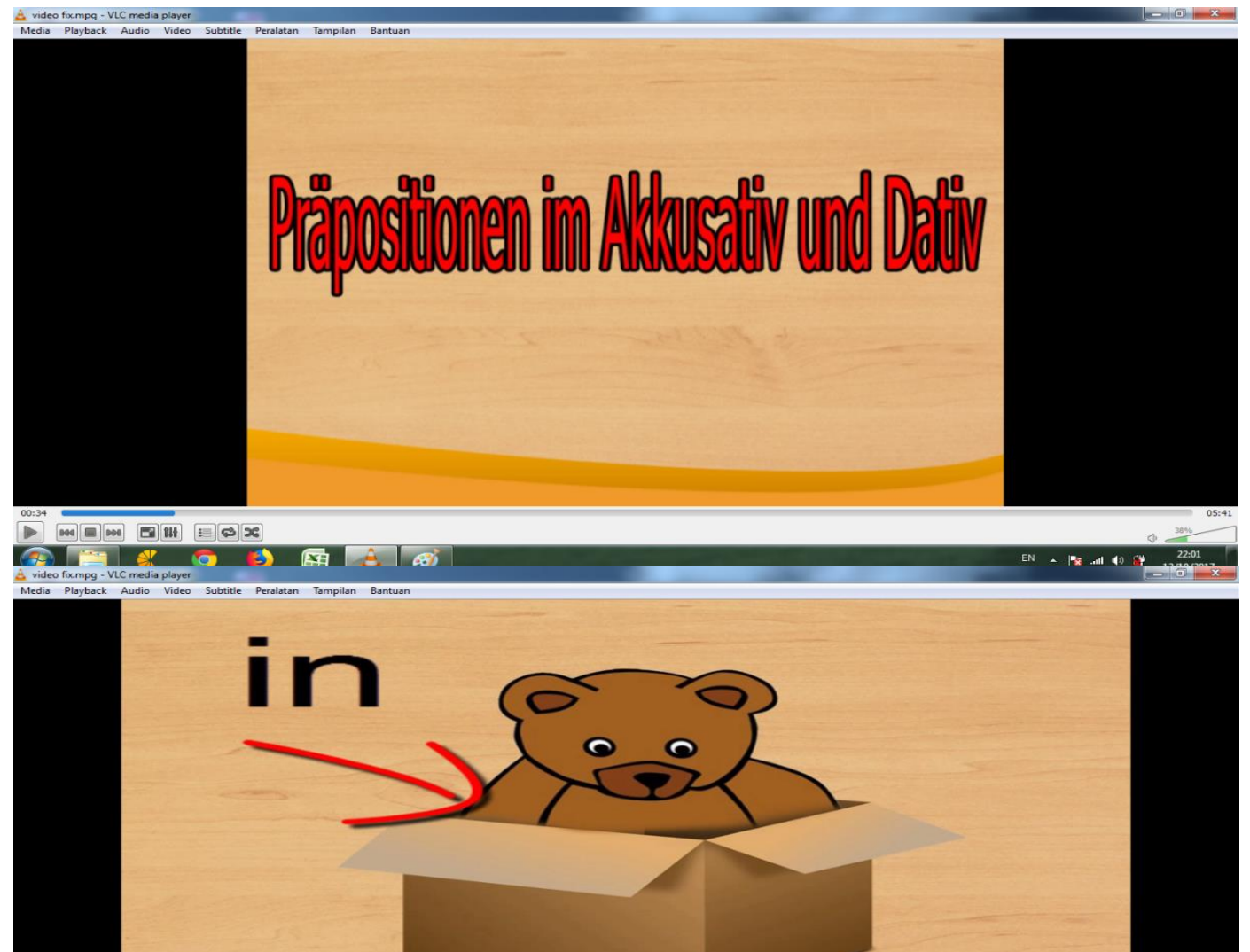
c) Ein Beispiel aus dem Ergebnis für den Satz Präpositionen Akkusativ und Dativ
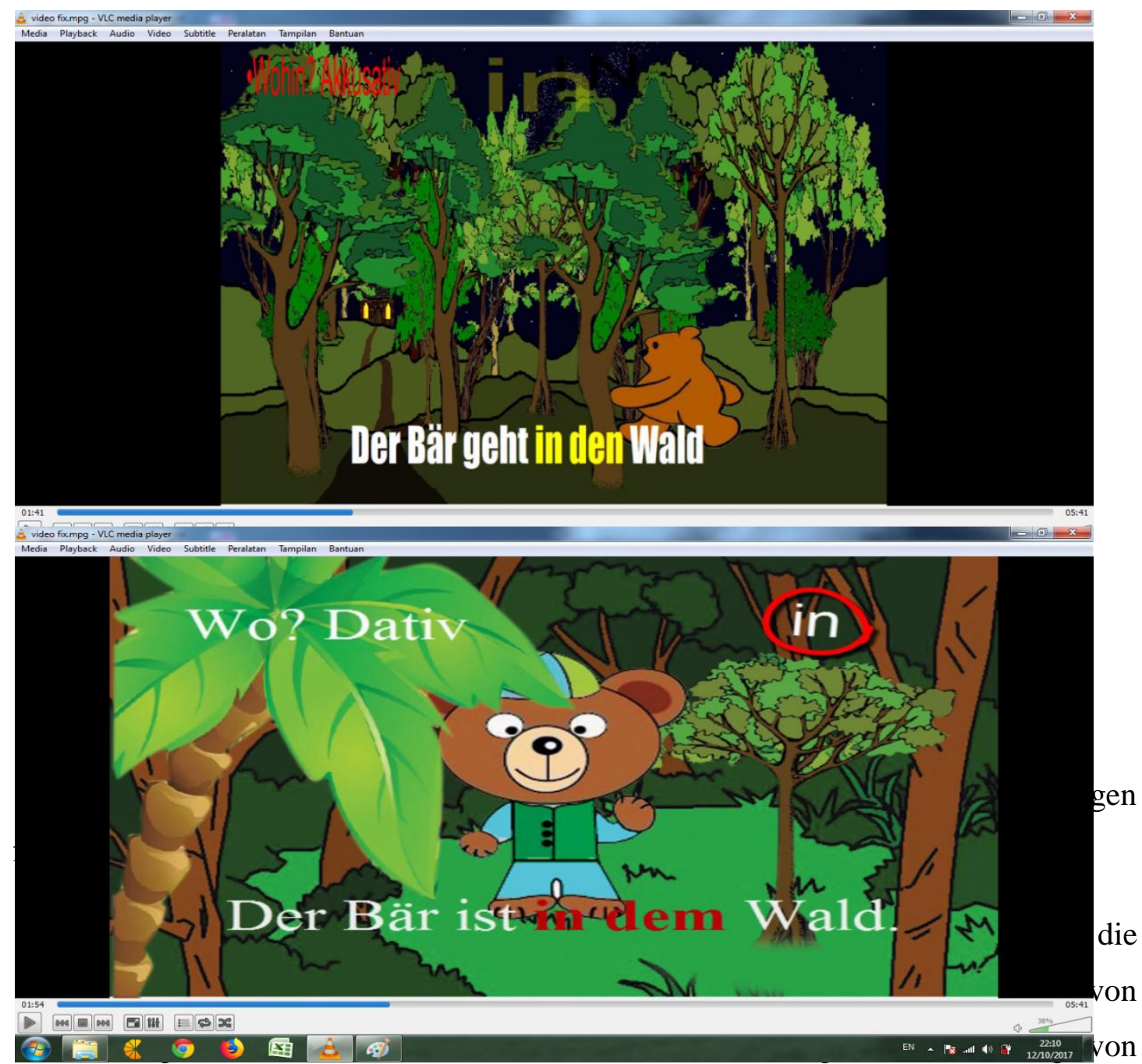

Information, (2) die Planung, (3) die Erstellung des Pdodukts, (4) die Validierung von Experten, (5) der Revision des Produkts.

2. Das Ergebnis der Erstellungeiner Animation als Lernmedium für die Grammatik im Deutschunterricht ist:

a. DieserUntersuchung hat eine Animation alsLernmediumfür die Grammatik imDeutschunterrichterstellt, die dieserAbschlussarbeitangehängtwird. 
b. Die Erstellungeiner Animation als Lernmedium für die Grammatik im Deutschunterricht wurde von Experten evaluiert. Die Evaluation umfasst die Evaluation der Wörter und der Sprache mit dem Thema „Präpositonen Akkusativ und Dativ.

\section{LITERATURVERZEICHNIS}

Barus, Ulian dan Suratno. 2015. Pemanfaatan Candi Bahal sebagai Media Pembelajaran Alam Terbuka Dalam Proses Belajar Mengajar. Medan: Perdana Mitra Handalan

Binanto, Iwan. 2010.Multimedia Digital Dasar Teori dan Pengembangannya. Yogyakatra: CV ANDI

Bunadi, Suriman dan Zeembry. 2007. Membuat Animasi Kartu Ucapan dengan Flash 8. Jakarta: PT Elex Media Komputindo

Buziek, Gerd, et al. 2000. Dynamische Visualisierung Grundlage und Anwendungensbeispiel für kartografische Animationen. Berlin: Springer

Daryanto. 2010. Media Pembelajaran. Bandung: Satu Nusa

Eppert, Franz. 2008. Grammatik-ABC für Deutsch als Fremdsprachen auf Zertifikatsniveau und Niveaustufen A1, A2, B1, B2. Frankfurt: R.G. Fischer

Fauziah, Annisa Nurul. 2014. Fehler Anlyse der Deutschen Grammatik in dem Aufsatz der Schüler XI IPA 2 SMA NEGERI 1 DRIYOREJO. Surabaya: Universitas Negeri Surabaya

Heller, Sabine. 2008. Charakter-Anmimation in Film und Fernsehen Analyse und Entwicklung von zwei und dreidimensionalen Charakteren. Nordersredt Germany: GRINN

MZ, Yumarlin. 2012. Pengembangan Multimedia Pembelajaran IPS untuk Siswa Sekolah Dasar. Sleman: Akademi Maritim Kompleks Pendidikan Wahidin

Sanjaya, Wina. 2012. Media Komunikasi Pembelajaran. Jakarta: Kencana Prenadanedia Grup 
Stang, Christian, et al. 2014.Rechtschreibung und Grammatik. Berlin: Dudenverlag

Susilana, Rudi dan Cepi Riyana. 2009. Media Pembelajaran. Bandung: CV Wacana Prima

Wahyono, Teguh. 2006. 36 Jam Belajar Komputer Animasi dengan Macromedia Flash 8. Jakarta: PT Elex Media Komputindo

http://ahmadfauzihidayat.blogspot.co.id/2013/03/modul-macromedia-flash-8.html diunduh pada tanggal 08 Juni 2017 pukul 11.00 WIB

https://isakabdillah.wordpress.com/author/isakabdillah/diunduh pada tanggal $20 \mathrm{Juli}$ 2017 pukul 20.00 WIB

https://sugitoorlando.com/2014/12/14/cara-penggunaan-macromedia-flash-8/ diunduh pada tanggal 20 Juli 2017 pukul 20.05 WIB

Vopy Endang Lestari is an alumni of German language study program of Unimed, Medan. 
Studia: Journal des Deutschsprogramms p-ISSN 2301-6108 e-ISSN 2654-9573

https://jurnal.unimed.ac.id/2012/index.php/studia/index 